\title{
BMJ Open Is the decline in neonatal mortality in northern Ghana, 1996-2012, associated with the decline in the age of BCG vaccination? An ecological study
}

\author{
Paul Welaga, ${ }^{1,2}$ Cornelius Debpuur, ${ }^{1}$ Peter Aaby, ${ }^{3}$ Abraham Hodgson, ${ }^{4}$ \\ Daniel K Azongo, ${ }^{1}$ Christine S Benn, ${ }^{2,5}$ Abraham Rexford Oduro ${ }^{1}$
}

To cite: Welaga P, Debpuur C, Aaby $\mathrm{P}$, et al. Is the decline in neonatal mortality in northern Ghana, 1996-2012, associated with the decline in the age of BCG vaccination? An ecological study. BMJ Open 2018;8:e023752. doi:10.1136/ bmjopen-2018-023752

- Prepublication history and additional material for this paper are available online. To view these files, please visit the journal online (http://dx.doi. org/10.1136/bmjopen-2018023752).

Received 22 April 2018 Revised 14 June 2018 Accepted 30 0ctober 2018

Check for updates

(c) Author(s) (or their employer(s)) 2018. Re-use permitted under CC BY-NC. No commercial re-use. See rights and permissions. Published by BMJ.

${ }^{1}$ Navrongo Health Research Centre, Navrongo, Ghana ${ }^{2}$ OPEN, Institute of Clinical Research, University of Southern Denmark, Denmark

${ }^{3}$ Bandim Health Project, Indepth Network, Bissau, Guinea-Bissau ${ }^{4}$ Research and Development Division, Ghana Health Service, Accra, Ghana

${ }^{5}$ CVIVA, Research Centre for Vitamins and Vaccines, Bandim Health Project, Statens Serum Institut, Copenhagen S, Denmark

Correspondence to

Paul Welaga;

pwelaga@yahoo.com

\section{ABSTRACT}

Objective To examine the association between early Bacille Calmette-Guerin (BCG) vaccination and neonatal mortality in northern Ghana.

Methods This ecological study used vaccination and mortality data from the Navrongo Health and Demographic Surveillance System. First, we assessed and compared changes in neonatal mortality rates (NMRs) and median BCG vaccination age from 1996 to 2012 . Second, we compared the changes in NMR and median BCG vaccination age from 2002 to 2012 by delivery place when data on delivery place were available.

Results Neonatal mortality rates declined from 46 to 12 per 1000 live births between 1996 and 2012 (trend test: $\mathrm{p}<0.001)$. Within the same period, median BCG vaccination age declined from 46 to 4 days (trend test: $p<0.001$ ). Among home deliveries, BCG vaccination age declined from 39 days in 2002 to 7 days in 2012 (trend test: $p<0.001$ ) and neonatal mortality declined by $24 / 1000$ (trend test: $p<0.001$ ). Among health facility deliveries, BCG vaccination age was stable around 3 days from 2002 to 2012 (trend test: $p=0.49$ ) and neonatal mortality declined by $9 / 1000$ (trend test: $p=0.04$ ). In a small study of children whose vaccination cards were inspected within the first 28 days of life, the HR for BCGvaccinated compared with BCG-unvaccinated children was 0.55 (95\% Cl 0.12 to 2.40$)$.

Conclusion The data support the hypothesis that early BCG vaccination may be associated with a decrease in neonatal mortality. However, as suggested by WHO, randomised control trials are required to address the question of whether there is indeed a causal association between early BCG vaccination and neonatal mortality.

\section{INTRODUCTION}

Bacille Calmette-Guerin (BCG) for tuberculosis (TB) control is one of the most widely used vaccines worldwide in low-income countries. ${ }^{1}$ The Expanded Programme on Immunization (EPI) in Ghana recommends that BCG be given at birth. ${ }^{2}$ However, due to the cost of vaccines, implementation challenges by health authorities as well as individual and household level factors, BCG vaccination is often delayed. ${ }^{3}$
Strengths and limitations of this study

- We used routine vaccination and survival data collected over a 17-year period from a large Health and Demographic Surveillance System site in Ghana.

- We assessed and compared changes in neonatal mortality rates and median Bacille Calmette-Guerin (BCG) vaccination age from 1996 to 2012 to assess the association between BCG vaccination age and neonatal mortality.

- This was mainly an ecological population-based study where exposures and outcomes were not directly linked.

- A small study using individual-level data to assess the association between BCG vaccination age and neonatal mortality was underpowered and under-reporting of BCG vaccination status and other limitations may have affected the accuracy of our estimates.

Observational studies and randomised control trials (RCTs) suggest that BCG vaccination may have non-specific survival benefits for children. ${ }^{4-7}$ In three RCTs among low-birthweight neonates in Guinea-Bissau, ${ }^{47}$ BCG vaccination was associated with a $38 \%$ $(95 \%$ CI $17 \%$ to $54 \%)$ ) reduction in neonatal mortality. The reduction in mortality was mainly due to fewer cases of neonatal sepsis and respiratory infections. Thus, BCG vaccination might have beneficial effects on child health outcomes in the first month of life that are not explained by protection against TB. Immunological studies have recently shown that BCG vaccines can train the innate immune system via epigenetic modifications at the monocyte level. ${ }^{8}$ This could possibly provide a mechanism whereby early BCG administration could modulate a child's ability to combat subsequent unrelated infections.

Even though child mortality is declining globally, millions of children still die every 
year before their fifth birthday and infections account for a large proportion of these deaths. ${ }^{9}$ More than $40 \%$ of these deaths occur within 28 days after birth (neonatal period), and this is a burden borne disproportionately by low-income and middle-income countries. While underfive mortality is declining, neonatal mortality appears to be declining at a much slower rate. ${ }^{10}$ There is therefore reason to examine whether better use of BCG could contribute to lower neonatal mortality.

In 2014, the WHO's Strategic Advisory Group of Experts on Immunization conducted a comprehensive review of the potential non-specific effects of vaccines and concluded that BCG may be associated with non-specific survival benefits. ${ }^{11}$ The WHO has been advocating for improved vaccination coverage levels. What is often not emphasised is the importance of timely administration of vaccines. To assess whether early BCG vaccination could be associated with a decrease in neonatal mortality, we examined the relationship between age at BCG vaccination and neonatal mortality using Health and Demographic Surveillance System (HDSS) data and routine vaccinations data of children from 1996 to 2012 in a mainly rural community in northern Ghana. We also examined the factors that are associated with the timing of BCG vaccination.

\section{MATERIALS AND METHODS Study setting}

The study was conducted in the Kassena-Nankana East municipal and Kassena-Nankana West district in the Upper East region of northern Ghana with an estimated population of about 160000 under continuous demographic surveillance. The study area covers a land area of $1675 \mathrm{~km}^{2}$ and lies between latitude $10.30^{\circ}$ and $11.10^{\circ}$ north and longitude $1.10^{\circ}$ west close to the Burkina Faso border. It has 1 main hospital (War Memorial Hospital) that serves as a referral hospital to nine clinics or health centres and 45 Community Health Compounds located mostly in rural communities and manned by trained nurses who provide basic healthcare as well as routine vaccinations. Previous analysis of causes of neonatal deaths in the study area showed that $32 \%$ of the causes were from infections, $21 \%$ from birth injury and asphyxia and $18 \%$ from prematurity, making these three the leading causes of neonatal deaths in the area. ${ }^{12}$

\section{Vaccination data}

Routine vaccination data from 1996 to 2012 collected by the Navrongo HDSS were used for the analyses. ${ }^{13} 14$ Between 1996 and 2010, vaccination data were collected once annually from health cards of children $<2$ years of age, except in 2001 when the HDSS failed to collect vaccination data. From 2011 to 2012, vaccination data were updated every 4 months for children aged 3 years or younger. The HDSS field teams visited all households 3-4 times a year during the period of the study from 1996 to 2012 to document demographic events such as new births, deaths and migrations. Data on educational attainment and household possessions are documented as part of the HDSS operations.

\section{BCG vaccine strains used in Ghana}

Even though Ghana introduced routine BCG vaccination into its EPI programme in 1978, accurate data on BCG strains used in Ghana from 1978 to 2006 are not available. ${ }^{15}$ The following BCG strains were used for vaccination in Ghana: 2007, BCG-Danish, produced by Danish Statens Serum Institute; 2008 to 2009, the BCG-Russia, produced by Bulbio; and from 2010 to 2012, BCG-Japan, produced by Japan BCG Laboratory. ${ }^{15}$

\section{Statistical methods}

Data were analysed using STATA V.12.1. To assess the association between median BCG vaccination age and neonatal mortality, we calculated yearly neonatal mortality rates (NMRs) per 1000 live births for children born in the study area from 1996 to 2012. We also calculated median BCG vaccination age by birth year from 1996 to 2012. Trends in NMR and median BCG vaccination age were assessed to identify any association for children delivered in health facilities and at home, respectively.

We carried out an individual-level analysis of children delivered at home and visited in the first 28 days of life using a Cox regression model to examine any association between their BCG vaccination status and subsequent neonatal mortality. We adjusted for socioeconomic status (wealth index), sex, maternal age, maternal education, interview year and season of birth. The wealth index was computed using principal component analysis from household assets as an estimate of household socioeconomic status. The household assets included several separate items, from large to medium assets (eg, land, car and motorbike ownership) to smaller household items (eg, radio, fan ownership). The children were categorised into BCG-vaccinated and BCG-unvaccinated based on BCG vaccination status in their health cards. Children with health cards and recorded BCG vaccination dates were classified as BCG-vaccinated. Those with no health card or no record of BCG vaccination on their vaccination card were categorised as BCG-unvaccinated. Children whose vaccination cards were not seen or those we did not meet at the time of the home visit were excluded from the substudy analysis. There was no loss to follow-up in the individual-level study. The exposure data (vaccination status) were collected before the occurrence of the outcome for all participants. The criterion for inclusion was that a child should first be visited and be alive at the time of the visit in the first 28 days after birth. It was only in subsequent visits that an outcome could be assessed. The exposure data (vaccination) and the outcome data (survival) were independently collected, in most cases, by different fieldworkers.

Virtually all deliveries in health facilities were vaccinated, and we did not have complete data on delivery place for the entire period of the study. Data on place of 
delivery were only available from 2002. In addition, other background characteristics including access to healthcare of the children delivered in health facilities, which we could not measure in this study, may be different from those delivered at home. We therefore limited the analysis to home deliveries, which are likely to have the same background characteristics including health-seeking behaviour and access to healthcare. To reduce survival bias, ${ }^{16}$ neonatal mortality was assessed prospectively using the landmark approach from the date of visit until the child was 28 days of age. Survival bias leads to differential misclassification of vaccine status because children who survive have better information than those who died during follow-up because health cards for dead children were often not available for inspection. With incomplete vaccine information for dead children, vaccination becomes automatically associated with a strong beneficial effect. In the landmark approach, only vaccine information collected on the date of visit is used, and the vaccine status becomes a time-fixed variable during follow-up in the analysis. This approach gives conservative estimates. ${ }^{16}$

\section{Patient and public involvement}

Patients were not involved in developing the hypothesis, the specific objectives or the research questions. They were also not involved in the design of the survey instruments or conduct of the study. No patients were involved in the interpretation of study results or write up of the manuscript. There are no plans to disseminate the results of the research to study participants or the relevant study community.

\section{RESULTS}

In all, we recorded 67431 live births and 1753 neonatal deaths (2.6\%) over the period from 1996 to 2012. Table 1 describes the demographic characteristics of neonates and mothers included in the population-based study. Within the period of the analysis, median BCG vaccination age declined from 46 days in 1996 to 4 days in 2012 (figure 1). The proportion of children vaccinated in day one after birth increased from 8\% in 1996 to 33\% in 2012 (table 2). In the same period, NMR declined from 46 per 1000 live births in 1996 to 12 per 1000 live births in 2012 (trend test: $\mathrm{p}<0.001$ ) (figure 1). In the first week of life, mortality for days 2-7 declined from 16/1000 to $7 / 1000$ (trend test $\mathrm{p}<0.001$ ); after the first week of life, the mortality declined to $1 / 8$, from $16 / 1000$ to $2 / 1000$ live births (trend test $\mathrm{p}<0.001$ ) (table 2 ).

During the period of this study, no neonatal death was recorded from TB. An analysis of the trends in the causes of neonatal deaths and the cause-specific mortality rates during different periods of the study from 1996 to 2012 showed that the decline in the cause-specific mortality rates for infections was stronger compared with prematurity and birth injury, particularly after the first week of life (online supplementary table 1 ).
Table 1 Demographic characteristics of neonates and mothers included in the population-based study in Navrongo Health and Demographic Surveillance System: 1996-2012

\begin{tabular}{|c|c|c|}
\hline Variable & Number & $\%$ \\
\hline \multicolumn{3}{|l|}{ Sex } \\
\hline Female & 33606 & 49.8 \\
\hline Male & 33825 & 50.2 \\
\hline \multicolumn{3}{|l|}{ Season of birth } \\
\hline Dry & 37411 & 55.5 \\
\hline Rainy & 30020 & 44.5 \\
\hline \multicolumn{3}{|l|}{ Year of birth } \\
\hline 1996 & 4222 & 6.3 \\
\hline 1997 & 4277 & 6.3 \\
\hline 1998 & 3828 & 5.7 \\
\hline 1999 & 4103 & 6.1 \\
\hline 2000 & 4352 & 6.5 \\
\hline 2001 & 4011 & 5.9 \\
\hline 2002 & 3967 & 5.9 \\
\hline 2003 & 4060 & 6.0 \\
\hline 2004 & 3923 & 5.8 \\
\hline 2005 & 3732 & 5.5 \\
\hline 2006 & 3744 & 5.6 \\
\hline 2007 & 3960 & 5.9 \\
\hline 2008 & 3640 & 5.4 \\
\hline 2009 & 3782 & 5.6 \\
\hline 2010 & 3910 & 5.8 \\
\hline 2011 & 3957 & 5.9 \\
\hline 2012 & 3963 & 5.9 \\
\hline \multicolumn{3}{|l|}{ Maternal education } \\
\hline No education & 27175 & 40.3 \\
\hline Primary & 21263 & 31.5 \\
\hline $\begin{array}{l}\text { Junior secondary school/ } \\
\text { middle school }\end{array}$ & 10667 & 15.8 \\
\hline Secondary & 4535 & 6.7 \\
\hline Tertiary & 1711 & 2.5 \\
\hline Missing & 2080 & 3.2 \\
\hline \multicolumn{3}{|l|}{ Maternal age } \\
\hline$<20$ & 8090 & 12.0 \\
\hline $20-34$ & 42971 & 63.7 \\
\hline $35-$ & 15743 & 23.4 \\
\hline Missing & 627 & 0.9 \\
\hline
\end{tabular}

\section{BCG vaccination age by place of delivery}

Table 3 describes the decline in median BCG vaccination age by place of delivery from 2002 when this information was first collected. The decline in age of BCG vaccination was a dual process. First, the proportion of health facility deliveries increased from $28 \%$ in 2002 to $88 \%$ in 2012 , 


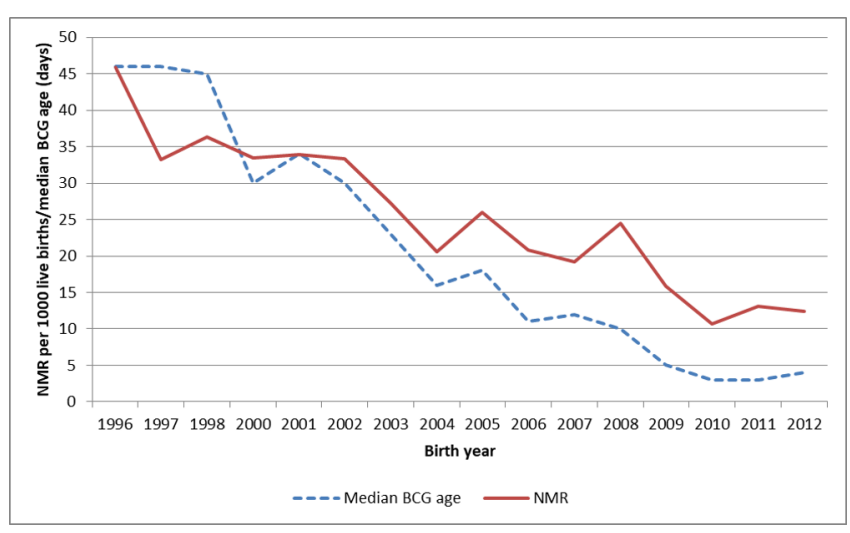

Figure 1 Trends in median Bacille Calmette-Guerin (BCG) vaccination age and neonatal mortality in Navrongo Health and Demographic Surveillance System: 19922012. NMR, neonatal mortality rate.

and these children were vaccinated early; the median BCG vaccination age in health facilities was around 3 days throughout the period. Second, the age at BCG vaccination decreased also among home deliveries, the median BCG vaccination age for home deliveries declined from 39 days in 2002 to 7 days in 2012 (table 3). Overall, the BCG coverage between children aged 2-23 months was high for both health facility and home deliveries throughout the period. BCG coverage for home deliveries increased from $89 \%$ in 2002 to $99 \%$ in 2012. Among health facility deliveries, BCG coverage was around 100\% throughout the period from 2002 to 2012.
Decline in NMR among home and health facility deliveries

The decline in BCG vaccination age and in NMR among home deliveries was much faster than what was observed among health facility deliveries (figure 2). In the 20022012 period, NMR among home deliveries declined by $24 / 1000$, that is, from $37 / 1000$ in 2002 to $13 / 1000$ live births in 2012 (table 3) (trend test: $\mathrm{p}<0.001$ ). For health facility deliveries, the decline in NMR was $9 / 1000$, that is, from 22/1000 in 2002 to 13/1000 live births in 2012 (trend test: $\mathrm{p}=0.04$ ). The decline in NMR among home deliveries was faster than among health facility deliveries, though the difference in their slopes was not statistically significant $(-1.85$ for home deliveries compared with -1.41 for facility deliveries, $\mathrm{p}=0.52$ ).

Since previous studies have suggested that BCG may have stronger beneficial effects for girls than for boys, ${ }^{17}{ }^{18}$ we examined the decline in NMR by sex (online supplementary table 2). Among home deliveries, the NMR for girls declined from 37/1000 in 2002 to 8/1000 live births in 2012 (trend test: $\mathrm{p}=0.007)$, whereas it declined from 37 per 1000 live births to 20 per 1000 live births for boys (trend test: $\mathrm{p}=0.26$ ). However, though the trend was significant in its own right among girls, but not among boys, there was no statistically significant difference between the two sexes (slope: -1.21 for girls compared with -0.56 for boys, $\mathrm{p}=0.29$ ). In the substudy with individual-level data, the HR for BCG-vaccinated boys versus BCG-unvaccinated boys was $0.92(0.20-4.25)$, and for girls, the HR was zero due to no death among the BCG-vaccinated girls; test of interaction $(\mathrm{p}=1.00)$.

Table 2 Median Bacille Calmette-Guerin (BCG) vaccination age and neonatal mortality rates (NMRs) in Navrongo Health and Demographic Surveillance System (HDSS): 1996-2012

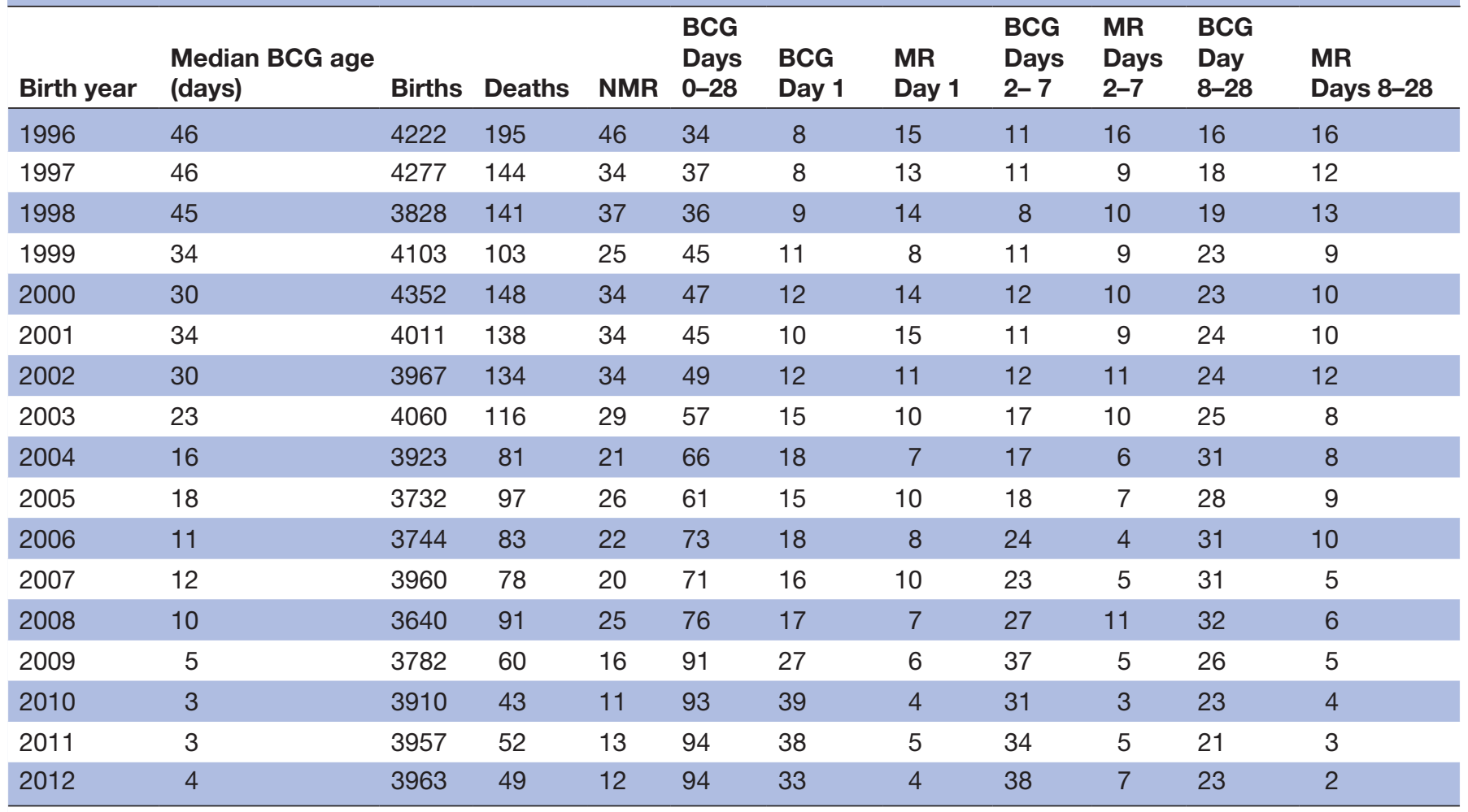

$B C G$, proportion $B C G$ vaccinated; $M R$, mortality rates. 
Table 3 Median Bacille Calmette-Guerin (BCG) vaccination age and neonatal mortality rates (NMRs) by place of delivery

\begin{tabular}{|c|c|c|c|c|c|c|c|c|c|}
\hline \multirow{3}{*}{$\begin{array}{l}\text { Birth } \\
\text { year }\end{array}$} & \multirow{3}{*}{$\begin{array}{l}\text { Proportion } \\
\text { delivered } \\
\text { in health } \\
\text { facility }\end{array}$} & \multicolumn{4}{|c|}{ Home delivery } & \multicolumn{4}{|c|}{ Health facility delivery } \\
\hline & & \multirow{2}{*}{$\begin{array}{l}\text { Median } \\
\text { BCG age }\end{array}$} & \multirow{2}{*}{$\begin{array}{l}\text { NMR } \\
0-28 \text { days }\end{array}$} & \multirow{2}{*}{$\begin{array}{l}\text { Proportion } \\
\text { BCG } \\
\text { vaccinated } \\
0-7 \text { days }\end{array}$} & \multirow{2}{*}{$\frac{M R}{\text { 8-28 days }}$} & \multirow[t]{2}{*}{$\begin{array}{l}\text { Median BCG } \\
\text { age }\end{array}$} & \multirow{2}{*}{$\begin{array}{l}\text { NMR } \\
0-28 \text { days }\end{array}$} & \multirow{2}{*}{$\begin{array}{l}\text { Proportion } \\
\text { BCG } \\
\text { vaccinated } \\
0-7 \text { days } \\
\end{array}$} & \multirow{2}{*}{$\begin{array}{l}\text { MR } \\
\text { 8-28 days }\end{array}$} \\
\hline & & & & & & & & & \\
\hline 2002 & 28 & 39 & 37 & 9 & 14 & 3 & 22 & 64 & 6 \\
\hline 2005 & 38 & 25 & 24 & 21 & 6 & 6 & 28 & 54 & 14 \\
\hline 2006 & 36 & 17 & 20 & 27 & 9 & 3 & 27 & 67 & 12 \\
\hline 2007 & 42 & 20 & 20 & 22 & 5 & 4 & 18 & 63 & 3 \\
\hline 2008 & 50 & 20 & 22 & 23 & 5 & 4 & 29 & 64 & 9 \\
\hline 2012 & 88 & 7 & 13 & 51 & 4 & 3 & 13 & 75 & 2 \\
\hline
\end{tabular}

MR, mortality rates.

\section{BCG vaccination status and subsequent neonatal mortality}

For the substudy of children seen in the first month of life, we examined whether BCG vaccination status was associated with subsequent neonatal mortality; 4910 children delivered at home and visited within the first 28 days after delivery were included in the analysis. There was under-reporting of BCG vaccination at the neonatal visits and the comparison was therefore not very precise; nearly $20 \%$ of the children classified as 'BCG-unvaccinated' at the first home visit were found to have been BCG-vaccinated before the first survey date using data from later follow-up visits (online supplementary table 3 ). This inconsistency in reporting is probably due to the cultural tradition of secluding the mother and newborn child during the first months after delivery. The neonatal mortality rate among BCG-unvaccinated children was 147/1000 compared with 81/1000 person-years for BCG-vaccinated children, the adjusted HR being $0.55(0.12-2.40)$ for BCG-vaccinated compared with BCG-unvaccinated (table 4 ).

\section{Effect of the health system on time to BCG vaccination}

Community-based Health Planning and Services

The Community-based Health Planning and Services (CHPS) became a national healthcare delivery policy in Ghana in the beginning of the new century. ${ }^{19}$ CHPS was originally tested in Navrongo from 1994 to 2003. The study was a quasi-experimental trial designed to test the relative impact of different strategies for introducing community health services in the communities. ${ }^{20}$ Trained nurses were relocated into rural communities to provide basic healthcare including routine vaccinations to children. The study area was divided into four experimental cells. Cell 1 had community health volunteers (CHVs) only, cell 2 had trained nurses called community health

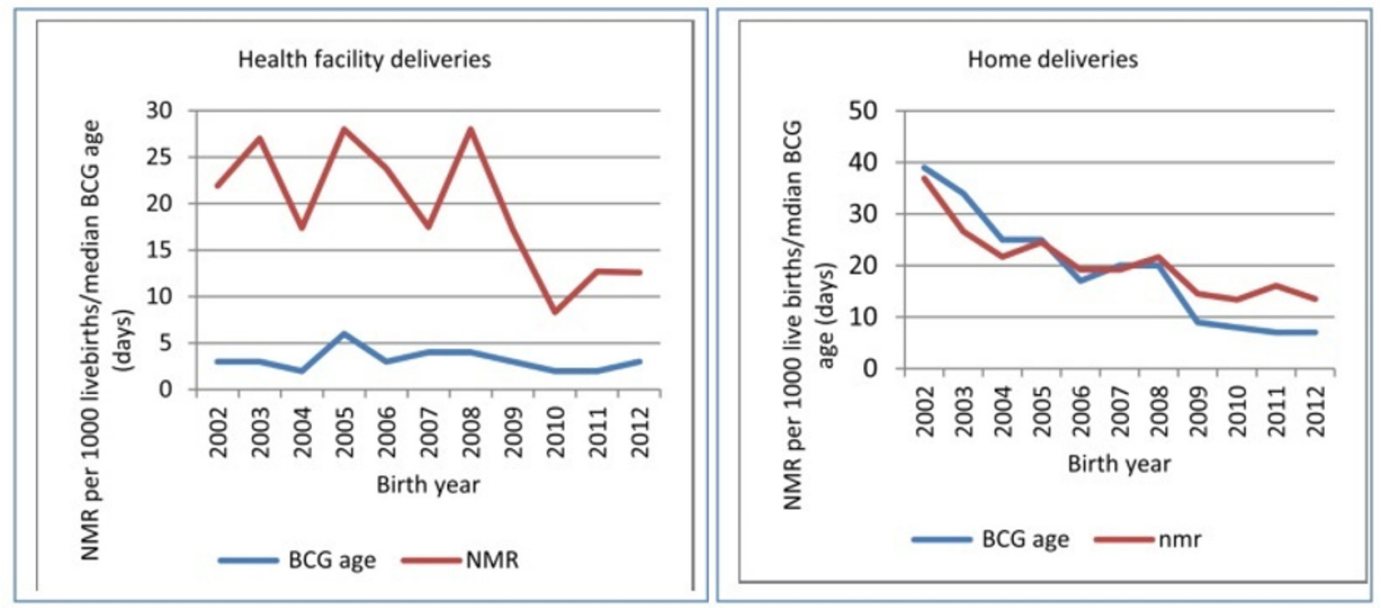

Figure 2 Trends in median Bacille Calmette-Guerin (BCG) vaccination age and neonatal mortality rates (NMRs) by home and health facility deliveries in Navrongo Health and Demographic Surveillance System: 2002-2012. 
Table 4 Mortality rates (MRs) and HRs from time of home visit up to 28 days of life among home deliveries

\begin{tabular}{|c|c|c|c|c|}
\hline Variable & Number (\%) & $\begin{array}{l}\text { MR/1000 person-years } \\
\text { (death/total) }\end{array}$ & HR (year adjusted) & HR (fully adjusted)* \\
\hline \multicolumn{5}{|c|}{ BCG status at time of home visit } \\
\hline BCG-unvaccinated & 4049 (82.5) & $147(26 / 177)$ & Ref & Ref \\
\hline BCG-vaccinated & $861(17.5)$ & $81(2 / 25)$ & $0.54(0.12-2.33)$ & $0.55(0.12-2.40)$ \\
\hline \multicolumn{5}{|l|}{ Maternal education } \\
\hline No education & $4147(84.5)$ & $135(23 / 170)$ & Ref & Ref \\
\hline Primary/JSS & $590(12.0)$ & $159(4 / 25)$ & $1.20(0.42-3.48)$ & $1.00(0.34-2.98)$ \\
\hline Secondary/tertiary & $154(3.1)$ & $174(1 / 6)$ & $1.12(0.15-8.31)$ & $1.41(0.19-10.55)$ \\
\hline Missing & $4(0.4)$ & $0(0 / 1)$ & & \\
\hline \multicolumn{5}{|l|}{ Sex of child } \\
\hline Male & $2440(49.7)$ & $170(17 / 100)$ & Ref & Ref \\
\hline Female & $2470(50.3)$ & $109(11 / 101)$ & $0.65(0.30-1.38)$ & $0.64(0.30-1.37)$ \\
\hline \multicolumn{5}{|l|}{ Wealth index } \\
\hline Poorest & $1718(35.0)$ & $155(11 / 71)$ & Ref & Ref \\
\hline Poorer & $1323(27.0)$ & $201(11 / 55)$ & $1.27(0.55-2.93)$ & $1.23(0.53-2.85)$ \\
\hline Poor & $1066(21.7)$ & $91(4 / 44)$ & $0.57(0.18-1.8)$ & $0.55(0.18-1.74)$ \\
\hline Less poor & $709(14.4)$ & $71(2 / 28)$ & $0.42(0.09-1.91)$ & $0.42(0.09-1.92)$ \\
\hline Least poor & $73(1.5)$ & $0(0 / 3)$ & - & - \\
\hline Missing & $21(0.4)$ & $0(0 / 1)$ & - & - \\
\hline \multicolumn{5}{|l|}{ Season of birth } \\
\hline Dry & $2643(53.8)$ & $120(13 / 108)$ & Ref & Ref \\
\hline Rainy & 2267 (46.2) & $161(15 / 93)$ & $1.18(0.56-2.50)$ & $1.20(0.57-2.54)$ \\
\hline \multicolumn{5}{|l|}{ Maternal age } \\
\hline$<20$ & $459(9.4)$ & $322(6 / 19)$ & $3.38(1.09-10.53)$ & $3.38(1.06-10.76)$ \\
\hline $20-34$ & 2989 (60.8) & $130(16 / 123)$ & Ref & Ref \\
\hline $37+$ & $1464(29.8)$ & $100(6 / 60)$ & $1.33(0.52-3.40)$ & $1.32(0.51-3.40)$ \\
\hline
\end{tabular}

${ }^{*}$ Adjusted for socioeconomic status (wealth index), sex, season of birth, maternal age, maternal education and interview year. BCG, Bacille Calmette-Guerin; JSS, Junior Secondary School.

officers (CHOs) only, cell 3 had both CHOs and CHVs, and cell 4 had no interventions.

As seen in table 5 , the decline in BCG vaccination age and the increase in the proportion of health facility delivery happened more quickly in the intervention communities compared with the non-intervention communities.

\section{BCG phial open policy}

Children in Ghana should receive BCG vaccination at birth. Even though health workers are expected to open a BCG phial if only one child is qualified to be vaccinated, this is often not done to reduce wastage. In the region where this study was conducted, in early 2009 the health authorities insisted on health workers to open a BCG phial even if only one child was present for vaccination. As seen in figure 3, there was a sharp increase in the proportion of children vaccinated in the first 7 days of life from $44 \%$ in 2008 to $65 \%$ in 2009 . Neonatal mortality also declined further in the same period.

\section{DISCUSSION}

BCG vaccination age declined remarkably from a median age of 46 days in 1996 to 4 days in 2012. In the same period, neonatal mortality rates also declined from 46 to 12 per 1000 live births. The decline in BCG vaccination age among home deliveries was much faster than health facility deliveries where BCG vaccination age remained fairly constant over the period. In parallel, the neonatal mortality decline was much faster for home deliveries compared with health facility deliveries. Though the individual-level analysis was of limited quality and provided a conservative estimate, because some children classified as 'unvaccinated' had received BCG already and more received BCG during follow-up, it also pointed to a reduced risk of neonatal death in BCG-vaccinated compared with BCG-unvaccinated children.

The decline in BCG vaccination age could be attributed to two processes; increasing proportions of women delivering at health institutions and the age at BCG vaccination decreasing among home deliveries. Children delivered 
Table 5 Age at Bacille Calmette-Guerin (BCG) vaccination and neonatal mortality rates (NMRs) comparing villages with community nurses and control villages with no intervention

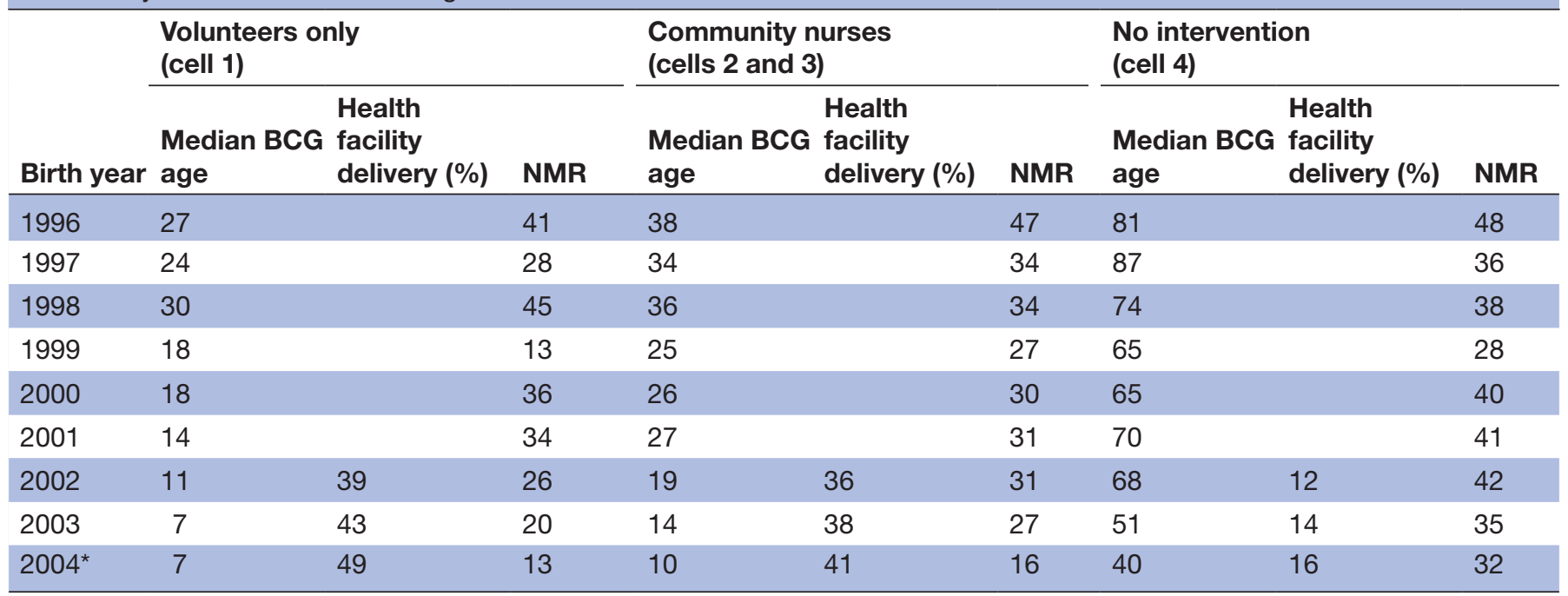

*From 2004 to 2005, the intervention (cell 3) of placing nurses in communities was extended to all the cells in the study area. Information place of delivery is only available from 2002.

in health facilities are expected to be vaccinated with BCG before being discharged. However, health workers sometimes refuse to open the BCG phial if there are few children in the facility for BCG vaccination. Instead, they rather recommend that mothers and children return on a scheduled date; on such dates, several children would then be present, and vaccine wastage would be reduced. In early 2009, the health authorities in the region instituted a policy to open the BCG phial even if it involved just one child (figure 3 ). This might have contributed to the decline in BCG vaccination age as children who were not even born in health facilities would have the opportunity to be vaccinated on first contact with a health facility. The CHPS programme, which was tested in the study area, also contributed to the decline in age at BCG vaccination. Community health officers and community health nurses resident in the community were able to visit mothers at home to deliver vaccines. Getting the

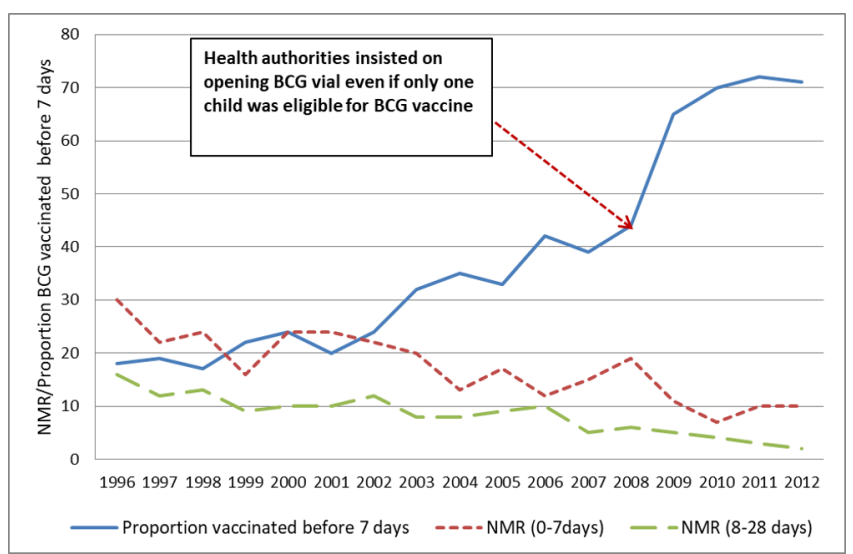

Figure 3 Proportion Bacille Calmette-Guerin (BCG) vaccinated at 0-7 days and neonatal mortality rates (NMRs) in Navrongo Health and Demographic Surveillance System: 1996-2012. vaccination programme to function efficiently to ensure that BCG vaccines are administered early after birth could possibly help to reduce neonatal mortality.

\section{Interpretation}

The parallel decline in mortality rates and BCG vaccination age could be a coincidence. However, there are several reasons why the results suggest that the decline in mortality rates over the period may be associated with the decline in BCG vaccination age.

First, neonatal mortality declined to almost $1 / 4$ from 46 to 12 per 1000 live births over the period of the analysis. The strongest decline occurred after the first week of life when the children might have received BCG. The decline was stronger for infections than for other causes of neonatal death.

Second, the decline was much faster among home deliveries, where the BCG vaccination age declined rapidly, compared with health facility deliveries, where BCG vaccination age remained the same around 3 days of life.

Third, the neonatal mortality rates showed some major declines from 1998 to 1999, 2002-2004; 2005-2006 and 2008-2010 (table 2). Notably, similar declines in BCG vaccination age were observed within the same periods.

Fourth, though based on small numbers, the individual-level analysis involving the small number of home deliveries seen alive and visited before they were 28 days supported that BCG vaccinated had a possible reduced risk of neonatal death compared with BCG-unvaccinated children. The individual-level analysis provided a direct measure of exposure and outcome, and the findings support what we observed at the population level. The survival analysis was done using a landmark approach to reduce survival bias. About $43 \%$ of the children classified as BCG-unvaccinated received BCG during the neonatal period using data from follow-up visits. Furthermore, we 
noted that there was under-reporting of BCG vaccination at home visits in the neonatal period. Culturally, mothers and their newborns in this study area are often kept away from the public and are most of the time indoors during the first month after delivery. We suspect fieldworkers who visited these households so early after delivery were most likely given convenient answers to prevent them from having access to the mother and the baby. We think this is most likely the reason for under-reporting of BCG vaccination in the first month of life.

Nonetheless, the effect size was similar to that observed in three RCTs in Bissau, where BCG vaccination was associated with a $38 \%$ reduction in neonatal mortality. ${ }^{4}$

The RCTs of BCG on neonatal mortality were done in low birthweight infants. Our population-based study supports a possible beneficial non-specific effect of BCG vaccination in normal-birthweight children in Africa. Kleinnijenhuis et al offered an immunological explanation for the beneficial non-specific effects of BCG by showing that BCG induces heterologous protection against unrelated pathogens via epigenetic reprogramming of monocytes. ${ }^{8}$

Our results should be interpreted with caution because exposures and outcomes were not directly linked, and it is difficult to separate BCG vaccination-related effects from other potential confounders and interventions. However, the findings are important in explaining the declining mortality patterns and for further investigation and intervention.

Other factors might have accounted for the decline in neonatal mortality rates over the period. Some of these factors include improvement in the healthcare delivery system (availability of better obstetric care, better primary healthcare services), demographic changes (fewer number of children per household), general improvement in the economy of the area (improved income levels, infrastructure, maternal education, employment), improvement in the nutritional status of neonates, etc.

This was largely an ecological study, and ecological fallacy may be a major challenge in making causal inference on the individual level in this study. In the small observational study of individual-level data, we were limited with respect to the number of confounders that we could adjust for due to lack of data. There are likely many unmeasured differences between the BCG vaccinated and unvaccinated children. For example, it is possible that BCG vaccinated children could have benefited from some form of care or counselling from health workers at the time of vaccination that this study could not document. We were also limited on the number of children included at the individual-level analysis due to the strict inclusion criteria of first visiting the child in the first 28 days of life. The likely uncontrolled confounders and low statistical power could have affected our risk estimates. In addition, the study included neonates from largely a rural population in Northern Ghana, which may limit the generalisability of the study results to other settings. It is unclear whether similar results would be observed in different settings.

There has not been any recorded policy to delay or withhold BCG vaccination from severely ill young neonates in the period of this study in the study area. Even though we could not assess the degree to which BCG vaccination was delayed or withheld, it was possible that some health workers or mothers could have decided to delay or withhold BCG vaccination due to severe illness, even though this is not an official policy. Therefore, the possibility of reverse causality as a possible partial explanation for the observed association between early BCG vaccination and neonatal mortality cannot be ruled out. However, the unvaccinated group should have become an increasingly select frail group as the vaccination coverage increased and the mortality rate among unvaccinated children should therefore have increased over time. This did not happen (online supplementary table 4), and we therefore do not think that reverse causality is the predominant explanation.

To address the question on whether BCG has non-specific beneficial effects on infant survival, SAGE established a working group on non-specific effects of BCG, DTP and measles vaccine. The WHO immunisation and vaccines-related implementation research advisory committee (IVIR-AC) concluded that RCTs were indeed required to resolve the question. ${ }^{21} \mathrm{WHO}$ recently circulated a call for public comments on draft protocols of clinical trials to assess any non-specific effects of BCG and measles vaccine. ${ }^{21} 22$

The WHO recommends BCG vaccination at birth. Funders should consider monitoring local implementation practices to ensure that wastage management of vaccines does not delay BCG vaccination. Individual health facilities may consider constructing a process map or use of continuous quality improvement methodology to learn about the barriers to timely administration of BCG to all eligible infants. BCG programmes are usually evaluated by vaccination coverage in children aged 12-23 months. Importantly, it is clear from the present study this evaluation would not capture the striking differences in the timing of BCG vaccination between health facility and home deliveries.

In conclusion, the present study supports a possible beneficial non-specific effect of early BCG vaccination on neonates and suggests that the tremendous reduction in neonatal mortality in northern Ghana during the last decades may partly be explained by efforts to decrease BCG vaccination age. However, as suggested by WHO, further RCTs may be required to address the question of whether there is indeed a causal association between early BCG vaccination and neonatal mortality.

Acknowledgements The authors thank Peter Wontuo, Felix Kondayire, George Wak and the rest of the field and computing staff of the Navrongo HDSS for collecting and processing the data. They also thank INDEPTH Network for coordinating some of the project activities. 
Contributors PW and PA proposed the present analysis. PW, CD, PA, AH, DA, CSB and ARO supervised the data collection. PW conducted the analysis and wrote the first draft of the paper. PW, CD, PA, ARH, DA, CSB and ARO contributed with comments and approved the final version of the paper.

Funding This work was supported by the Navrongo Health Research Centre, DANIDA (grant: 104.Dan.8-920) and European Union FP7 support for OPTIMUNISE (grant: Health-F3-2011-261375).

Competing interests None declared.

Patient consent for publication Not required.

Ethics approval The study was reviewed and approved by the institutional review board of the Navrongo Health Research Centre.

Provenance and peer review Not commissioned; externally peer reviewed.

Data sharing statement № additional data are available.

Open access This is an open access article distributed in accordance with the Creative Commons Attribution Non Commercial (CC BY-NC 4.0) license, which permits others to distribute, remix, adapt, build upon this work non-commercially, and license their derivative works on different terms, provided the original work is properly cited, appropriate credit is given, any changes made indicated, and the use is non-commercial. See: http://creativecommons.org/licenses/by-nc/4.0/.

\section{REFERENCES}

1. Dye C. Making wider use of the world's most widely used vaccine: Bacille Calmette-Guerin revaccination reconsidered. J $R$ Soc Interface 2013;10:20130365.

2. Profile: Ghana's expanded programme on immunization in ghana. http://www.who.int/countries/gha/publications/EPI_Profile.pdf (Assessed 17 Dec 2017).

3. Olusanya BO. Pattern and determinants of BCG immunisation delays in a sub-Saharan African community. Health Res Policy Syst 2010;8:1.

4. Aaby $P$, Roth $A$, Ravn $\mathrm{H}$, et al. Randomized trial of BCG vaccination at birth to low-birth-weight children: beneficial nonspecific effects in the neonatal period? J Infect Dis 2011;204:245-52.

5. Kabir Z. Is all-cause mortality a useful epidemiological endpoint in vaccine trials? An example of BCG (Bacille-Calmette-Guerine). Int J Epidemiol 2003:32:161-2.

6. Roth A, Jensen H, Garly ML, et al. Low birth weight infants and Calmette-Guérin bacillus vaccination at birth: community study from Guinea-Bissau. Pediatr Infect Dis J 2004;23:544-50.

7. Biering-Sørensen S, Aaby P, Lund N, et al. Early bcg-denmark and neonatal mortality among infants weighing $<2500 \mathrm{~g}$ : A randomized controlled trial. Clin Infect Dis 2017;65:1183-90.
8. Kleinnijenhuis J, Quintin J, Preijers F, et al. Bacille Calmette-Guerin induces NOD2-dependent nonspecific protection from reinfection via epigenetic reprogramming of monocytes. Proc Natl Acad Sci U S A 2012;109:17537-42.

9. Liu L, Johnson HL, Cousens S, et al. Global, regional, and national causes of child mortality: an updated systematic analysis for 2010 with time trends since 2000. Lancet 2012;379:2151-61.

10. Oestergaard MZ, Inoue M, Yoshida S, et al. Neonatal mortality levels for 193 countries in 2009 with trends since 1990: a systematic analysis of progress, projections, and priorities. PLoS Med 2011;8:e1001080.

11. Higgins JP, Soares-Weiser K, López-López JA, et al. Association of BCG, DTP, and measles containing vaccines with childhood mortality: systematic review. BMJ 2016;355:i5170.

12. Welaga $\mathrm{P}$, Moyer CA, Aborigo R, et al. Why are babies dying in the first month after birth? A 7-year study of neonatal mortality in northern Ghana. PLoS One 2013;8:e58924.

13. Ross D, Dollimore N, Smith P, et al. Vitamin A supplementation in northern Ghana: effects on clinic attendances, hospital admissions, and child mortality. Ghana VAST Study Team. Lancet 1993;342:7-12.

14. Binka FN, Bawah AA, Phillips JF, et al. Rapid achievement of the child survival millennium development goal: evidence from the Navrongo experiment in Northern Ghana. Trop Med Int Health 2007;12:578-83.

15. Phillips RO, Phanzu DM, Beissner M, et al. Effectiveness of routine $B C G$ vaccination on buruli ulcer disease: a case-control study in the Democratic Republic of Congo, Ghana and Togo. PLoS Negl Trop Dis 2015;9:e3457.

16. Jensen H, Benn CS, Lisse IM, et al. Survival bias in observational studies of the impact of routine immunizations on childhood survival. Trop Med Int Health 2007;12:5-14.

17. Aaby P, Nielsen J, Benn CS, et al. Sex-differential effects on mortality of BCG and diphtheria-tetanus-pertussis vaccines in a rural area with high vaccination coverage: observational study from Senegal. Trans $R$ Soc Trop Med Hyg 2016;110:527-33.

18. Aaby $P$, Vessari $\mathrm{H}$, Nielsen $\mathrm{J}$, et al. Sex differential effects of routine immunizations and childhood survival in rural Malawi. Pediatr Infect Dis J 2006;25:721-7.

19. Nyonator FK, Awoonor-Williams JK, Phillips JF, et al. The Ghana community-based health planning and services initiative for scaling up service delivery innovation. Health Policy Plan 2005;20:25-34.

20. Binka FN, Nazzar A, Phillips JF. The Navrongo Community Health and Family Planning Project. Stud Fam Plann 1995;26:121-39.

21. World Health Organization. Immunization, vaccines and biologicals. http://www.who.int/immunization/research/implementation/nse_ protocol_comments/en (Assessed 17 Dec 2017).

22. World Health Organization: http://www.who.int/immunization/ research/implementation/Synopsis_BCG_Study_protocol_2_April_17. pdf?au=1 (Assessed 17 Dec 2017) 